\title{
Women in Mathematics
}

\section{A Review by Katie Spurrier Quertermous}

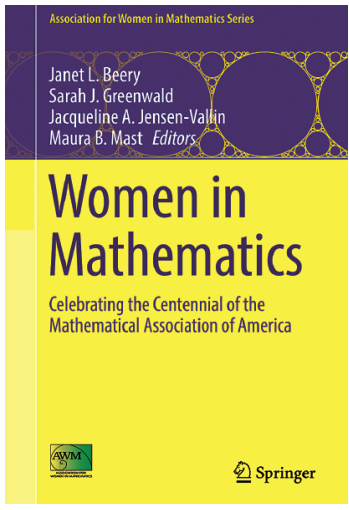

Women in Mathematics:

Celebrating the Centennial

of the Mathematical Association

of America

Janet L. Beery, Sarah J. Greenwald,

Jacqueline A. Jensen-Vallin, and

Maura B. Mast, editors

Springer International

Publishing, 2017

ISBN: 978-3319666938

In the last three years, ${ }^{1}$ at least three mass market books-Margot Lee Shetterly's Hidden Figures,

Nathalia Holt's Rise of the Rocket Girls, and Liza Mundy's Code Girls-and a major motion picture-Hidden Figures, based on Shetterly's book-have captivated audiences with the previously overlooked stories of women mathematicians who worked as human computers and cryptographers for the United States government. These works have offered the public a glimpse into the ongoing efforts of mathematicians and historians to write a social history of the lived experiences and contributions of women in the mathematical sciences. ${ }^{2}$ Women in Mathematics adds to this literature with a collection of twenty-one engaging articles that include biographies, historical and cultural studies, and profiles of outreach and education initiatives related to women in mathematics. Most of the articles in this col-

Katie Spurrier Quertermous is an associate professor of mathematics and statistics at James Madison University. Her email address is querteks@ jmu. edu.

${ }^{1}$ This number of years is correct through April 5, 2019.

${ }^{2}$ Margaret Murray describes the concept of social history in more detail in Chapter 5 of Women in Mathematics.

Communicated by Notices Book Review Editor Stephan Ramon Garcia.

For permission to reprint this article, please contact: reprint -permission@ams .org.

DOI: https://dx.doi.org/10.1090/noti 1861 lection are written for a broad mathematical audience that includes students.

This volume grew out of a contributed paper session at MAA MathFest 2015 that was sponsored by the Association for Women in Mathematics (AWM). In connection with the celebration of the one hundredth anniversary of the Mathematical Association of America, the session sought to "recognize the contributions, achievements, and progress of women mathematicians over the past 100 years" through "talks about mathematics done by women and historical or biographical presentations celebrating women in mathematics." As the editors note in their preface, the resulting collection of articles is a mix of current scholarship and exposition on a wide variety of topics related to women in mathematics as opposed to a balanced study of the participation of women in mathematics during this time. Some of the articles summarize or extend work that has appeared previously, including Judy Green and Jeanne LaDuke's detailed documentary history of all of the American women who earned PhDs in mathematics from American and European universities between 1886 and 1939 and Margaret Murray's research on American women who earned PhDs in mathematics between the years 1940 and 1959. As a result, the volume also serves as a survey of a portion of the existing literature and compellingly invites the reader to delve deeper into that work.

The first two parts of the book are dedicated to telling the stories of women mathematicians in articles that range in style from formal historical and cultural studies to personal reflections and collections of interviews. These articles include more than eighty biographical profiles of women mathematicians and statisticians as well as numerous more concise descriptions of the experiences and contributions of women in these fields. The profiles are a mix of short sketches grouped within larger discussions of the mathematical and social context of a particular time, place, or culture and more in-depth studies of the professional and personal lives of individual women. Most of the profiles 
focus on women from the United States, Canada, and Europe whose career paths are connected to academia or secondary education during at least a portion of their professional careers. Several chapters highlight women from underrepresented groups in mathematics, although the total number of biographical profiles of women from these groups is still small. The authors can be commended for including profiles of women whose stories are not widely known, so readers should expect to encounter at least a few unfamiliar names in these pages.

By presenting many of the profiles in groups, the articles emphasize both the connections between the individual women and the diversity of professional paths that they pursued even in times of limited career options. In their article on Girton College, Cambridge, Shawnee McMurran and James Tattersall profile a group of ten women who studied at Girton between 1880 and 1900 and achieved honors on the Mathematical Tripos exam despite the fact that women could not earn degrees from Cambridge at this time. These women applied their mathematical training to achieve success in numerous areas. As examples, Charlotte Angas Scott and Hertha Ayrton, the founders of Girton's Mathematical Club, had widely recognized research careers, Scott in algebraic geometry and Ayrton in engineering. Kate Knight Gale taught for many years, eventually becoming coowner and joint headmistress of a school in South Africa, and Margaret Frances Evans was Mathematical Mistress of St Leonards School before ending her professional career to focus on family life. Emily Perrin and Beatrice Mabel Cave-Browne-Cave were both computers in Karl Pearson's statistical research lab at University College, London. In the 1930s, Girton College's Yarrow Research Fellowship supported the early work of Olga Taussky-Todd and Mary Lucy Cartwright, who both became prolific research mathematicians, Taussky-Todd in matrix theory and number theory and Cartwright in function theory and differential equations. Cartwright was elected Mistress of Girton College in 1948, and she led the college in this role for nineteen years while continuing her active involvement in the mathematical community.

Erica Walker explores the history of Black women in mathematics in the United States in a reflective essay that draws upon research she conducted for her 2014 book, Beyond Banneker: Black Mathematicians and the Paths to Excellence. As part of this essay, Walker juxtaposes the stories of Euphemia Lofton Haynes (PhD 1943) and Evelyn Boyd Granville (PhD 1949), the first two Black women to be awarded doctorates in mathematics in the United States. ${ }^{3}$ Although they were born thirty-four years apart, both women were raised in Washington, DC, attended the same segregated high school, now called Dunbar High School, and earned undergraduate degrees from Smith College.

\footnotetext{
${ }^{3}$ Marjorie Lee Browne also finished the requirements for a PhD in mathematics in 1949, but her degree was not awarded until 1950.
}

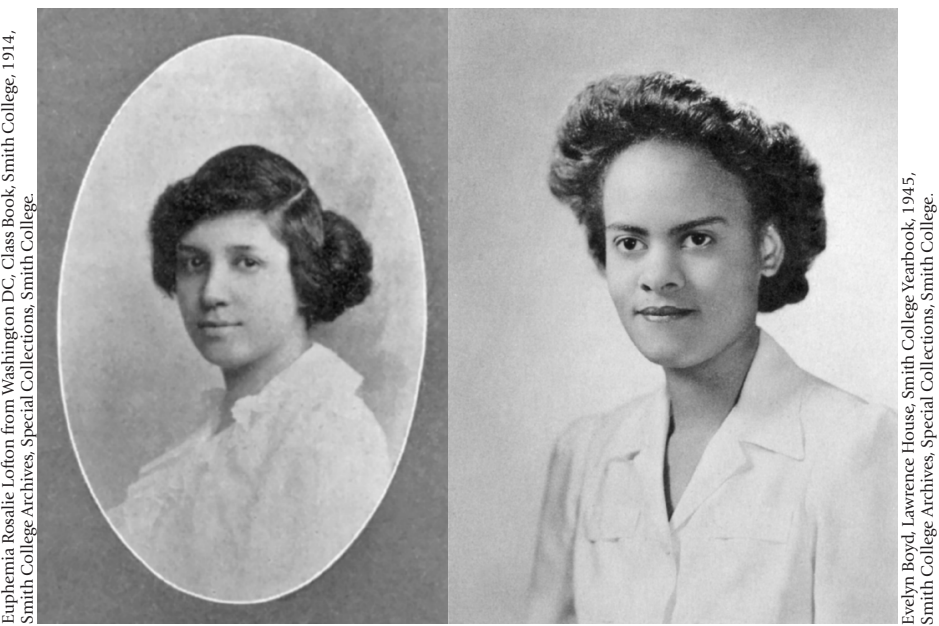

Figure 1. Euphemia Lofton Haynes (Smith College Class of 1914), left, and Evelyn Boyd Granville (Smith College Class of 1945), shown here in their college yearbook photos, attended the same Washington, $\mathrm{DC}$, high school and the same college before earning PhDs in mathematics.

Dunbar was known for having committed, highly educated teachers who encouraged their students to attend college. The influence of these teachers seems to have played a strong role in both Haynes's and Granville's enrollments at Smith. Despite their many commonalities, Granville has said that she did not learn about Haynes until 1999, almost twenty years after Haynes's death. Haynes taught in the DC public school system, established the mathematics department at Miner Teachers College (now part of the University of the District of Columbia), served as president of the DC Board of Education, and was a key advocate for integration of the DC public schools. Granville's career in industry at IBM and other NASA contractors was bookended by academic positions, including faculty positions at Fisk University and the University of Texas at Tyler. Continuing the chain of influence begun by the teachers at Dunbar, two Black women taught by Granville, Etta Zuber Falconer and Vivienne Malone-Mayes, earned PhDs in mathematics and mentored additional generations of mathematicians.

The celebration of women's often under-recognized contributions in areas such as mentoring, teaching, professional service, academic administration, and advocacy is a theme that runs throughout most of the articles. It is impossible to categorize all of these contributions here, so I will just mention a few that I learned about for the first time while reading this book. An article by Emelie Agnes Kenney explores the vital roles that women played in keeping mathematics alive in Nazi-occupied Poland by studying and teaching in the clandestine education system despite the danger they faced if caught. Kenney recounts how Irena Gołąb disguised her math classes as crochet circles to avoid detection. Patti Hunter discusses Gertrude Cox's efforts to support statistics training around the world, efforts that are 
not as widely known as her achievements in building statistics programs in the United States or her service as president of the American Statistical Association. As part of this work, Cox was a program specialist at Cairo University's Institute of Statistical Studies and Research in 1964-1965. She was a strong advocate for the importance of statistical consulting and personally consulted on numerous projects in Cairo. Norma Hernandez earned a PhD in mathematics education in 1970 and was a faculty member at the University of Texas at El Paso for thirty years, serving as dean of the College of Education for six of those years. Hernandez was born and raised in El Paso, and Luis Ortiz-Franco investigates how Hernandez's life experiences in this multicultural city likely influenced her research on the relationships between culture and mathematics in the context of the K-12 mathematics education of Latinx students.

Woven throughout the historical accounts of women's contributions are discussions of some of the challenges the women faced in their pursuit of mathematical careers, especially those they encountered prior to the 1970s. Each woman's story is different, but common obstacles include barriers to advanced training, bias against women in hiring and promotion practices, and a lack of recognition of women's accomplishments. Many of these obstacles reflect the prevailing social norms of the women's times, and this context is important for helping readers understand the significance of individual and collective contributions as well as the dedication and perseverance of the women who made these contributions.

Multiple authors comment anecdotally on the shifts in mathematical culture that have occurred during their lifetimes, but a formal analysis of these changes is not the focus of this volume. Readers are, however, offered glimpses into a few of the advocacy efforts that championed changes in culture. Jacqueline Dewar describes outreach activities to encourage middle and high school girls to study mathematics that grew out of regional organizing meetings of the AWM during the 1970s. Laura Turner explores discussions at Canadian Mathematical Society meetings in the late 1980s and early 1990s that highlighted the underrepresentation of women on journal editorial boards and as plenary lecturers at meetings, and Sue Geller discusses skits presented at the Summer and Winter Joint Mathematics Meetings from 1990 to 1994 that used humor to draw attention to micro-inequities.

The third part of the book focuses on outreach and educational efforts. In a joint article, Jacqueline Dewar and Sarah Greenwald outline courses they have developed on women and mathematics that combine history, mathematical work, and equity issues and suggest opportunities for readers to experiment with these ideas through shorter-term outreach activities. Karl Schaffer describes the process of creating Daughters of Hypatia, a full-length dance performance that shares the stories of historical and contemporary women in mathematics, showcases mathematical thinking embedded in arts and crafts, and challenges stereotypes through dance and music. In an example of the connections between articles, Daughters of Hypatia includes an adaption of one of Sue Geller's skits on micro-inequities mentioned above. Sylvia Bozeman, Susan D'Agostino, and Rhonda Hughes discuss the EDGE Program (Enhancing Diversity in Graduate Education), which supports women mathematicians from diverse backgrounds through an annual summer session for beginning graduate students and ongoing mentoring networks. As part of this article, the authors describe aspects of the program that are designed to increase students' ability to successfully navigate the academic, cultural, and social transitions they will encounter in graduate school.

Readers with a wide variety of mathematical, educa-

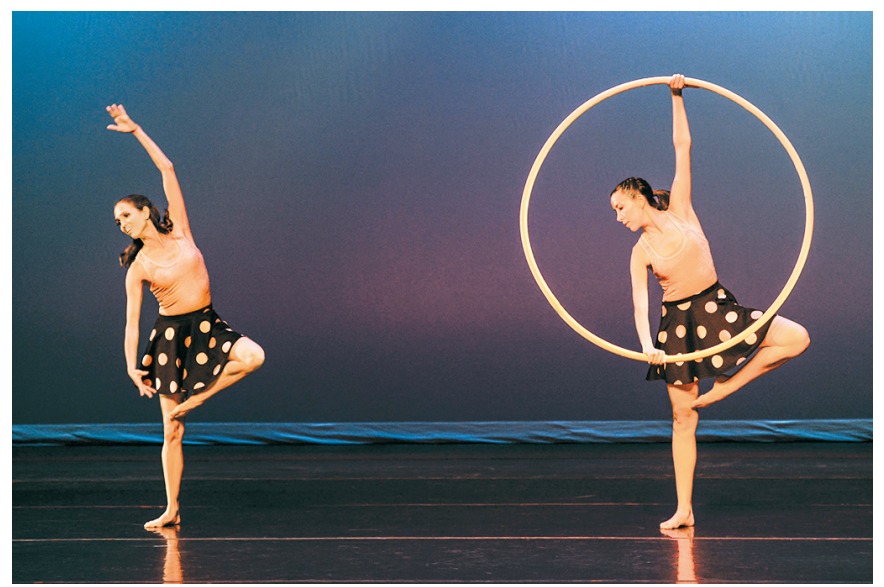

Figure 2. Daughters of Hypatia uses dance to share the stories of women mathematicians. In this 2015 photo, Laurel Shastri, left, and Lila Salhov perform "A Circle Has No Sides," one of several pieces that employ circular imagery.

tional, and historical interests will find the articles in this collection engaging and inspiring. Most of the articles are accessible to undergraduate and graduate students although a faculty mentor may be required at times to help students contextualize the importance of contributions described in terms of journal titles and professional committees. The material is well suited for inclusion in existing courses and math club activities, and it provides opportunities not only to teach students about the history of women in mathematics but also to introduce them to important elements of mathematical culture through stories in which women play central roles. For example, Amy Shell-Gellasch's article on Mina Rees provides an excellent starting point for discussing both research funding and the value of conference attendance. Rees was instrumental in shaping federal funding of mathematics research through her work with the US government's Applied Mathematics Panel and the Office of Naval Research, and this article recounts, in Rees's own words, the importance she placed on the personal connections and broad understanding of 
the current mathematical landscape that she established at conferences as preparation for these positions.

I highly recommend reading Women in Mathematics. The articles are compellingly written and contain a wealth of information about the lives and accomplishments of women mathematicians and the efforts of women and men to share this history and advocate for inclusion and diversity. They encourage readers to reflect on the experiences and contributions of women mathematicians, not only those profiled in the volume but also those in the readers' own communities. The articles challenge readers to celebrate the achievements, recognize the challenges, and use both to inform their understanding of the history and culture of mathematics. This volume invites us to continue learning about the fascinating past and present of women in mathematics and provides inspiration as we think about the future.

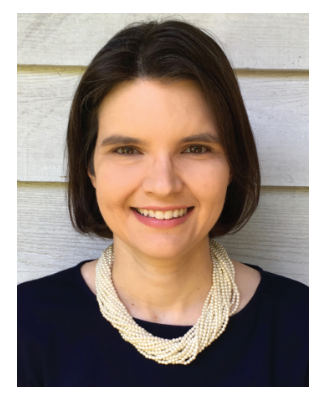

Katie Spurrier

Quertermous

\section{Credits}

Figure 1 photos are from the Smith College Archives, Special Collections, Smith College.

Figure 2 photo is by Clark Scott.

Author photo is courtesy of Katie Spurrier Quertermous.

Model and dimension reduction in uncertain and dynamic systems

January 27 - May 1, 2020

ORGANIZING COMMITTEE

Yanlai Chen, University of Massachusetts, Dartmouth

Serkan Gugercin, Virginia Tech

Misha Kilmer, Tufts University

Yvon Maday, Université Pierre et Marie Curie

Shari Moskow, Drexel University

Akil Narayan, University of Utah

Daniele Venturi, University of California, Santa Cruz

\section{PROGRAM DESCRIPTION}

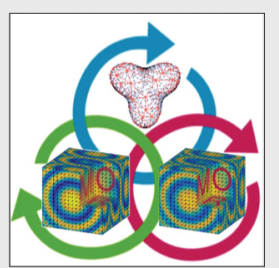

Today's computational and experimental paradigms feature complex models along with disparate and, frequently, enormous data sets. This necessitates the development of theoretical and computational strategies for efficient and robust numerical algorithms that effectively resolve the important features and characteristics of these complex computational models. The desiderata for resolving the underlying model features is often application-specific and combines mathematical tasks like approximation, prediction, calibration, design, and optimization. Running simulations that fully account for the variability of the complexities of modern scientific models can be infeasible due to the curse of dimensionality, chaotic behavior or dynamics, and/or overwhelming streams of informative data.

This program will integrate diverse fields of mathematical analysis, statistical sciences, data and computer science, and specifically attract researchers working on model order reduction, data-driven model calibration and simplification, computations and approximations in high dimensions, and data-intensive uncertainty quantification.

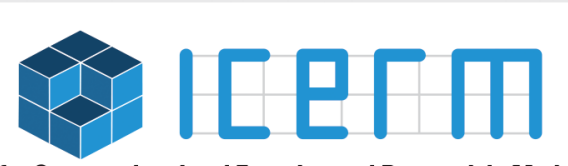

Institute for Computational and Experimental Research in Mathematics

Proposals being accepted: Semester Program

Topical/Hot Topics Workshops

Small Group Research Program

Summer Undergrad Program

ICERM is a National Science Foundation Mathematics Institute at Brown University in Providence, Rl.
Appications being accepted: Semester Program or Workshop Postdoctoral Fellowship Sponsorships being accepted: Academic or Corporate

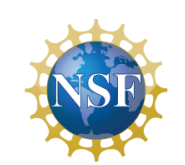

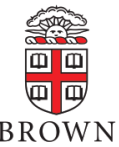

\section{icerm.brown.edu}

